\title{
Gaussian Mixture Model Based Damage Evaluation for Aircraft Structures
}

\author{
Qiuhui Xu, Shenfang Yuan*, Yuanqiang Ren \\ Research Center of Structural Health Monitoring and Prognosis, State Key Lab of Mechanics \\ and Control of Mechanical Structures, Nanjing University of Aeronautics and Astronautics, \\ Nanjing, 210016, P. R. China \\ qhx@nuaa.edu.cn, ysf@nuaa.edu.cn, renyuanqiang@nuaa.edu.cn,
}

Keywords: Structural Health Monitoring, Guided Wave Features, Aircraft Fatigue Test, Multi-Dimensional Gaussian Mixture Model, Migration Index

\begin{abstract}
The Guided Wave (GW) based Structural Health Monitoring (SHM) method is of significant research interest because of its wide monitoring range and high sensitivity. However, there are still many challenges in real engineering applications due to complex time-varying conditions, such as changes in temperature and humidity, random dynamic loads, and structural boundary conditions. In this paper, a Gaussian Mixture Model (GMM) is adopted to deal with these problems. Multi-dimensional GMM (MDGMM) is proposed to model the probability distribution of GW features under time-varying conditions. Furthermore, to measure the migration degree of MDGMM to reveal the crack propagation, research on migration indexes of the probability model is carried out. Finally, the validation in an aircraft fatigue test shows a good performance of the MDGMM.
\end{abstract}

\section{Introduction}

Structural Health Monitoring (SHM) technology has been gradually developed from basic theoretical research in the laboratory to practical aircraft engineering validations and applications ${ }^{[1,2]}$. The Guided Wave (GW) based SHM method has been widely studied due to its wide monitoring range and being sensitive to various types of small damage ${ }^{[3]}$. However, the real inservice conditions of aircraft structure are very complex, with many time-varying factors such as environmental temperature and humidity, random dynamic load and vibration, changing structural boundary condition, noise, and possibly others. The problem of reliable damage evaluation under time-varying conditions has become one of the main obstacles for applying SHM technology to real aircraft structures. These time-varying factors introduce uncertainty effects on GW signal features, leading to lower reliability and stability of SHM techniques in engineering applications.

In recent years, SHM methods based on probability and statistics are gradually studied to deal with the time-varying problem ${ }^{[4]}$. As an effective probability and statistics tool for characterizing uncertainties of GW signals, the Gaussian mixture model (GMM) has been gradually adopted for damage diagnosis of aerospace structure. Tschöpe and Wolff ${ }^{[5]}$ studied the basic GMM for damage degree classification on simple plate-like structures. However, they did not consider time-varying conditions. Banerjee et al. ${ }^{[6]}$ adopted GMM to classify the crack length of a platelike repaired composite specimen. The sudden change of the environmental temperature was considered as a time-varying factor in the validation of this method but it is a simple situation. Yuan and Qiu et al. ${ }^{[7]}$ proposed an enhanced dynamic GMM-based damage monitoring method considering the ordinary expectation-maximization algorithm can easily lead to a local optimal solution and a singular solution. The method was validated using the case of hole-edge crack 
monitoring of an aluminum plate and a real aircraft wing spar. All the research mentioned above has proved the potential to apply the GMM to solve the time-varying problem. However, deep research is still needed to be performed concerning the GMM for damage evaluation of real aircraft structures under time-varying conditions.

In this article, a Multi-Dimensional GMM(MDGMM) based damage monitoring method is proposed to deal with the uncertainties caused by time-varying conditions under the complex real in-service environment of an aircraft structure. MDGMM is proposed to model the probability distribution of the GW features under time-varying conditions. Furthermore, to measure the migration degree of MDGMM to reveal the damage propagation, the research on migration indexes (MI) of the probability mixture model is also carried out. Finally, validations in an aircraft fatigue test are presented which show a good performance of the MDGMM.

\section{Aircraft fatigue test and GW signals}

\section{Setup of the aircraft fatigue test}

The monitoring hotspot is located on the inner surface of the aircraft tail. Through visual inspection, the crack continues to grow during the whole aircraft fatigue test. During the different experimental days, the temperature range is $6.8^{\circ} \mathrm{C}$ to $14.7^{\circ} \mathrm{C}$.

A five-cycle sine burst modulated by the Hanning window with the central frequency of 150 $\mathrm{kHz}$ is used as the GW excitation signal. The fatigue test lasts 21 days and GW signals are collected during application of the dynamic load. Typical GW signals are shown in Fig. 1. Considering that the crack propagation path is near the direct propagation path of the $\mathrm{GW}$, the direct wave is the main signal segment for crack propagation monitoring. Thus, the length of the signal segment which is adopted to monitor the crack propagation is from $0.75 \times 10^{-4} \mathrm{~s}$ to $1.0 \times 10^{-}$ ${ }^{4} \mathrm{~S}$.

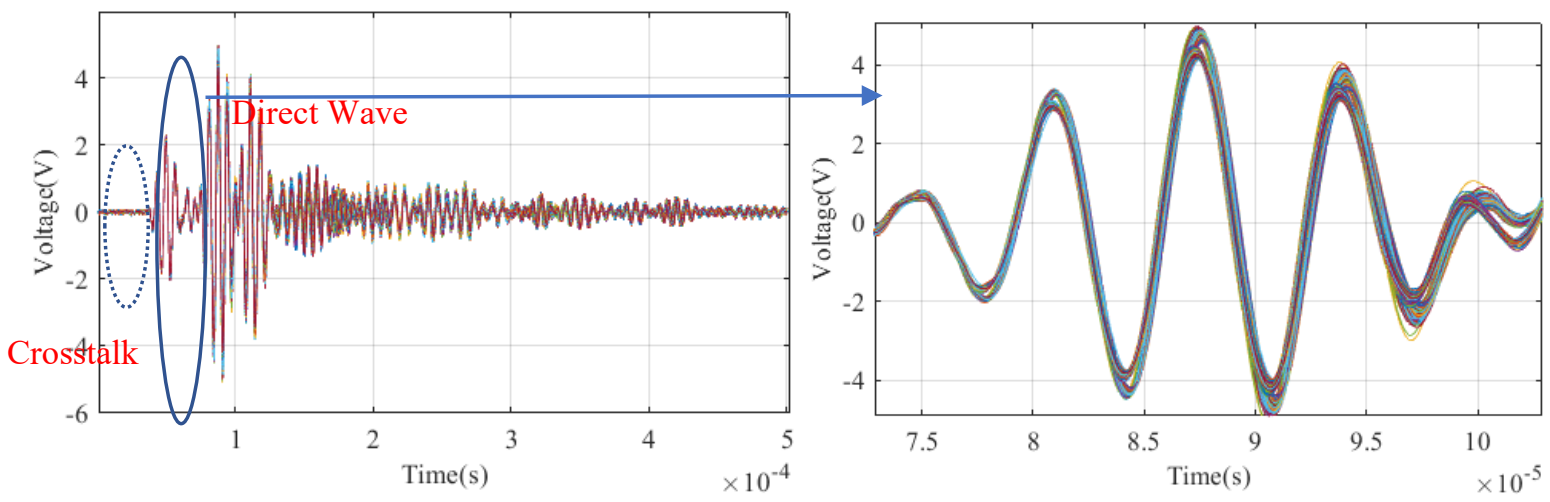

Figure 1. GW signals acquired under time-varying condition

\section{GW feature extraction}

For crack propagation monitoring by only one GW pitch-catch channel, a damage index (DI) can be used. This DI is used to measure the difference between the monitoring GW signal and the baseline GW signal. In this paper, multi-dimensional $D I$ s are selected to represent the damage information of the structure. $D I_{1}$ is the time-domain cross-correlation. $D I_{2}$ is the spectrum magnitude difference and $D I_{3}$ is the normalized correlation moment ${ }^{[8]}$.

The DIs during the fatigue test are shown in Fig.2. In conventional DI based SHM methods, a $D I$ threshold is often predefined. When the value of the $D I$ exceeds the threshold, a damage is assumed to have occurred. However, the variation of GW features introduced by time-varying conditions is random and complex. The evaluation of crack propagation is also difficult to be realized based on the results shown in Fig.2. 


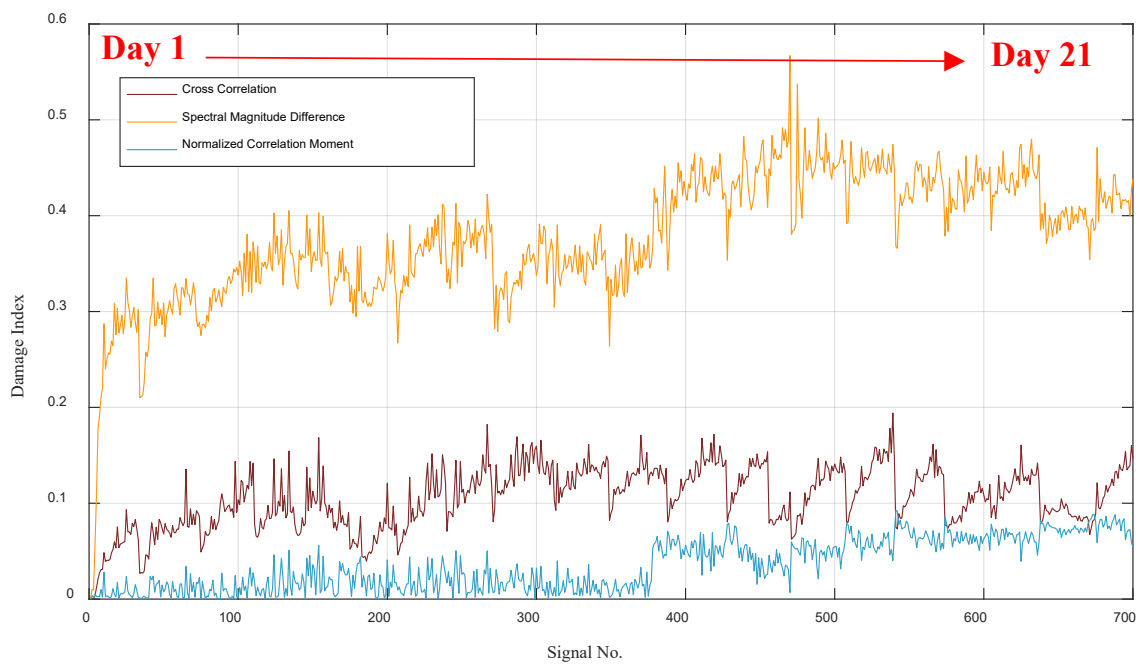

Figure 2. DIs under the dynamic load

\section{GW-MDGMM based damage evaluation method}

\section{Damage monitoring principle of the MDGMM}

The GW signals acquired under time-varying conditions can be considered as a mixture of uncertain changes. Consequently, the $\mathrm{GW}$ feature can be considered as a random variable. Let $\boldsymbol{X}=\left[\boldsymbol{X}_{1}, \boldsymbol{X}_{2}, \ldots \boldsymbol{X}_{k}, \ldots, \boldsymbol{X}_{K}\right]$ be a GW feature sample set composed by $K$ features that are obtained from $K \mathrm{GW}$ signals. $\boldsymbol{X}_{\boldsymbol{k}}$ denotes a $d$-dimensional $\mathrm{GW}$ feature in the sample set, where $\boldsymbol{X}_{\boldsymbol{k}}=\left[D I_{1}\right.$, $\left.D I_{2}, \ldots, D I_{d}\right]^{\mathrm{T}}$ and $k=1,2, \ldots K$. When a monitored structure is in the healthy state, the GW feature sample set only contains the uncertain influence introduced by time-varying conditions. Under this situation, the feature sample set can be regarded as a GW baseline feature sample set.

Assuming that $\boldsymbol{X}$ follows a finite mixed distribution, the mixture probability distribution $\xi(X)$ can be approximately modeled by a Gaussian mixture model (GMM). The GMM is expressed as Eq. 1.

$$
\xi(\boldsymbol{X} \mid \boldsymbol{\mu}, \boldsymbol{\Sigma})=\sum_{i=1}^{C} w_{i} \xi_{i}\left(\boldsymbol{X}_{k} \mid \boldsymbol{\mu}_{i}, \boldsymbol{\Sigma}_{i}\right)
$$

Where $C$ is the number of Gaussian components, $i=1,2, \ldots, C$. An important step is to determine the number of Gaussian components before modeling. $w_{i}$ is the mixture weight of the $i$ th Gaussian component. $\boldsymbol{\mu}_{i}$ and $\boldsymbol{\Sigma}_{i}$ are the mean and the covariance matrix of the $i$ th Gaussian component. The probability density function of each Gaussian component is a multi-dimensional (d-dimensional) Gaussian function which is expressed as Eq. 2.

$$
\xi_{i}\left(\boldsymbol{X}_{k} \mid \boldsymbol{\mu}_{i}, \boldsymbol{\Sigma}_{i}\right)=\frac{1}{(2 \pi)^{\frac{d}{2}} \sqrt{\left|\boldsymbol{\Sigma}_{i}\right|}} e^{-\frac{1}{2}\left(\boldsymbol{X}_{k}-\boldsymbol{\mu}_{i}\right)^{T} \boldsymbol{\Sigma}_{i}^{-1}\left(\boldsymbol{X}_{k}-\boldsymbol{\mu}_{i}\right)}
$$

The expectation-maximization $(E-M)$ algorithm is often used to construct the MDGMM ${ }^{[7]}$. First, the initial value of $\boldsymbol{\mu}_{i}, \boldsymbol{\Sigma}_{i}$, and $w_{i}$ can be obtained by the $k$-means clustering algorithm. Second, the $E$-step and the $M$-step are performed iteratively to obtain the optimized $\boldsymbol{\mu}_{i}, \boldsymbol{\Sigma}_{i}$, and $w_{i}$. 


\section{On-line migration measuring of the MDGMM}

The migration measuring result between the on-line monitoring MDGMM and the baseline MDGMM is defined as migration index (MI). Accompanying the damage propagation, two GMMs will be separated gradually. In this paper, Euclidean distance ${ }^{[9]}$ (E-distance), Mahalanobis distance ${ }^{[10]}$ (M-distance), Bhattacharyya distance ${ }^{[11]}$ (B-distance), and KL divergence ${ }^{[12]}$ are studied.

The Euclidean distance based model migration index can be calculated by Eq. 3 .

$$
d\left(\xi_{i}(0) \| \xi_{j}(n)\right)=\left(\boldsymbol{\mu}_{i}(0)-\boldsymbol{\mu}_{j}(n)\right)^{T}\left(\boldsymbol{\mu}_{i}(0)-\boldsymbol{\mu}_{j}(n)\right)
$$

The Mahalanobis distance based model migration index can be calculated by Eq. 4 .

$$
d\left(\xi_{i}(0) \| \xi_{j}(n)\right)=\left(\boldsymbol{\mu}_{i}(0)-\boldsymbol{\mu}_{j}(n)\right)^{T}\left(\frac{\boldsymbol{\Sigma}_{i}(0)+\boldsymbol{\Sigma}_{j}(n)}{2}\right)^{-1}\left(\boldsymbol{\mu}_{i}(0)-\boldsymbol{\mu}_{j}(n)\right)
$$

The Bhattacharyya distance based model migration index can be calculated by Eq. 5 .

$$
d\left(\xi_{i}(0) \| \xi_{j}(n)\right)=\frac{1}{8}\left(\boldsymbol{\mu}_{i}(0)-\boldsymbol{\mu}_{j}(n)\right)^{T}\left(\frac{\boldsymbol{\Sigma}_{i}(0)+\boldsymbol{\Sigma}_{j}(n)}{2}\right)^{-1}\left(\boldsymbol{\mu}_{i}(0)-\boldsymbol{\mu}_{j}(n)\right)+\frac{1}{2} \ln \frac{\left|\left(\boldsymbol{\Sigma}_{i}(0)+\boldsymbol{\Sigma}_{j}(n)\right) / 2\right|}{\left|\boldsymbol{\Sigma}_{i}(0)\right|^{1 / 2}\left|\boldsymbol{\Sigma}_{j}(n)\right|^{1 / 2}}
$$

The K-L divergence based model migration index can be calculated by Eq. 6 .

$$
d\left(\xi_{i}(0) \| \xi_{j}(n)\right)=\frac{1}{2} \operatorname{tr}\left[\boldsymbol{\Sigma}_{j}(n)^{-1} \boldsymbol{\Sigma}_{i}(0)+\left[\boldsymbol{\mu}_{j}(n)-\boldsymbol{\mu}_{i}(0)\right]^{T} \boldsymbol{\Sigma}_{j}(n)^{-1}\left[\boldsymbol{\mu}_{j}(n)-\boldsymbol{\mu}_{i}(0)\right]-d-\ln \left(\frac{\left|\boldsymbol{\Sigma}_{j}(n)\right|}{\left|\boldsymbol{\Sigma}_{i}(0)\right|}\right)\right]
$$

Where $\boldsymbol{\mu}_{i}(0), \boldsymbol{\Sigma}_{i}(0)$, and $w_{i}(0)$ are the mean, the covariance matrix, and the mixture weight of the $i$-th Gaussian component of $\boldsymbol{\xi}(0)$, respectively. $\boldsymbol{\mu}_{j}(n), \Sigma_{j}(n)$, and $w_{j}(n)$ are the mean, the covariance matrix, and the mixture weight of the $j$-th Gaussian component of $\xi(n)$, respectively. $\operatorname{tr}[\cdot]$ is the matrix trace.

Also, Eq. 7 is the result of considering the difference of the mixture weight between two Gaussian components.

$$
D\left(\xi_{i}(0) \| \xi_{j}(n)\right)=\sum_{i=1}^{K} w_{i}^{0} \min _{j=1}^{K}\left(d\left(\xi_{i}(0) \| \xi_{j}(n)\right)+\ln \frac{w_{i}(0)}{w_{j}(n)}\right)
$$

\section{The MDGMM based damage evaluation process}

When a new GW feature is obtained during an on-line damage monitoring process, a migration method can be adopted to rebuild the MDGMM. Once the on-line migrated MDGMM is obtained, the measuring methods are adopted to determine the crack occurrence.

\section{Crack propagation monitoring based on the GW-MDGMM}

The first $50 \mathrm{GW}$ features that are obtained from $50 \mathrm{GW}$ signals are used as a $\mathrm{GW}$ baseline sample set to construct a baseline MDGMM in the first part shown in Fig.3(a). All GW features are input one by one to calculate the MI. The number of Gaussian components is set to be 3 to cover the GW feature sample set. The baseline MDGMM is shown in Fig.3 (a). Based on the 
migration method, some typical on-line migrated MDGMMs accompanying the crack propagation are shown in Fig.3 (b)-(d) respectively.

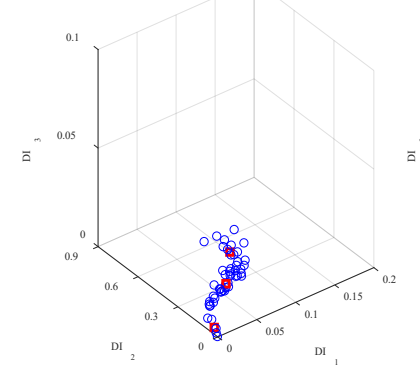

(a) Baseline MDGMM

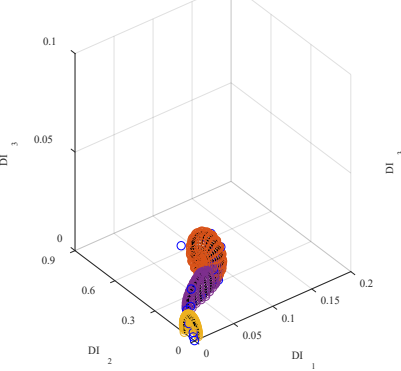

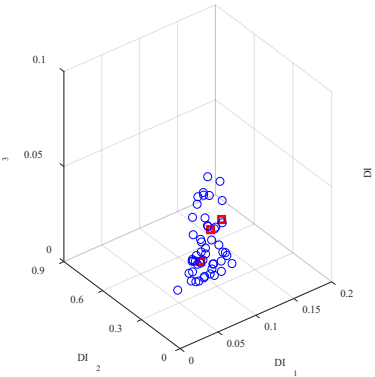

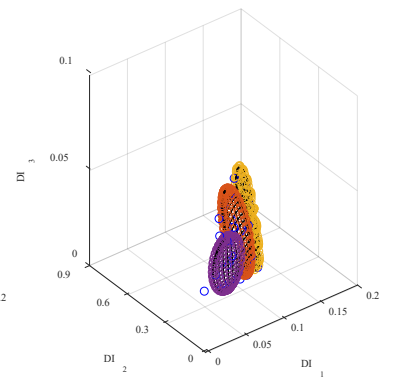

(b) On-line MDGMM (Phase

\section{1)}

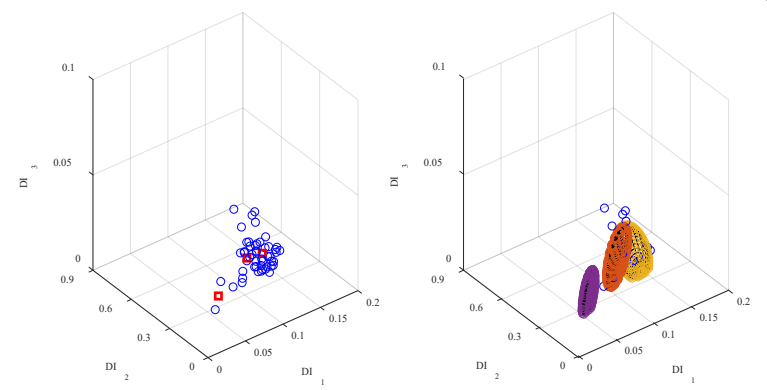

(c) On-line MDGMM (Phase 2)

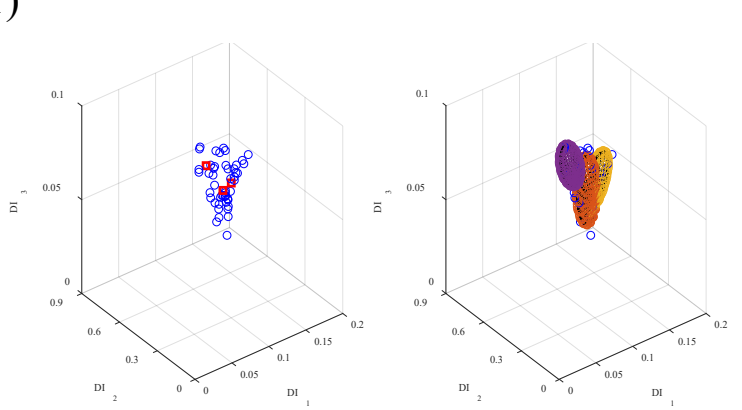

(d) On-line MDGMM (Phase 3)

Figure 3. MDGMM migration process

The MIs calculated by Eq. 3-6 are displayed in Fig.4. Compared to the DIs shown in Fig.2, the four MIs increase gradually and present a cumulative progressive trend that accompanies the crack growth under fatigue dynamic load. Therefore, a threshold can be easily defined to determine the crack occurrence, and the initial crack propagation can be reliably evaluated depending on the cumulative variation trend of the MIs.

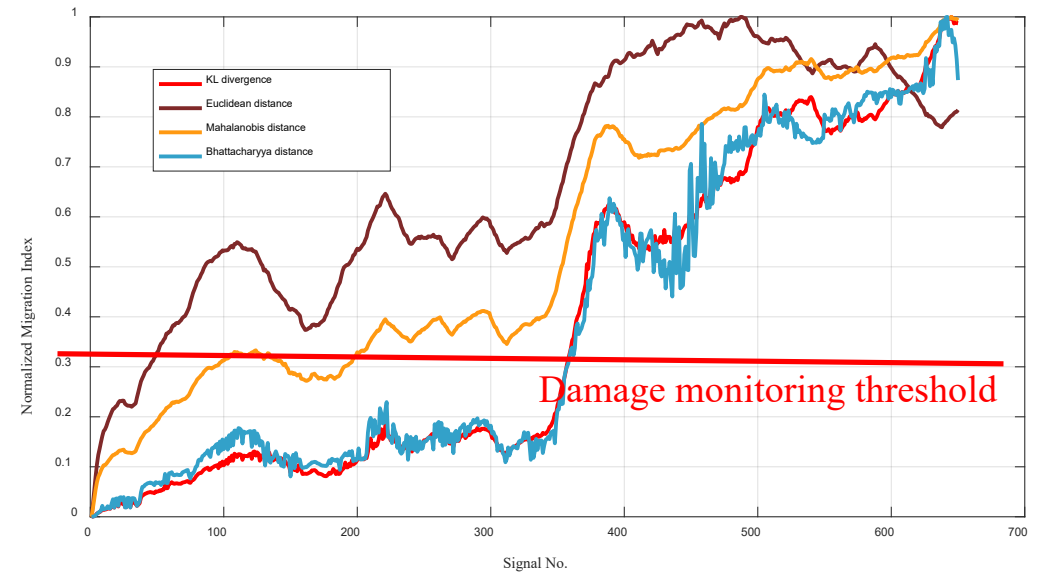

Figure 4. Crack propagation monitoring results

The overall trend of the four MIs is consistent, which indicates that these four distance methods can be used to measure the migration of MDGMM to a certain extent. As shown in Fig.4, the fluctuation of E-distance is large. After 400 monitoring times, E-distance drops 
sharply, showing that the reliability and accuracy of E-distance are the lowest. This may be because the E-distance only considers the mean difference but ignores the correlation between samples. The results show that KL divergence and B-distance are similar in measuring MDGMM migration distance. However, the volatility of B-distance is large and its stability is not good. In the early stage of damage monitoring, M-distance shows a larger value, which indicates that the sensitivity of M-distance is relatively high. In summary, M-distance and KL divergence show better performance in migration measuring of MDGMM.

\section{Conclusion}

An MDGMM based damage monitoring method is proposed in this article to improve crack propagation monitoring reliability under time-vary conditions. The monitoring results in an aircraft fatigue test show a good performance of the MDGMM. The whole method is a datadriven based probability and statistics method, with no mechanical models of damage and structure needed.

However, there are several further studies to be performed in the near future. As mentioned before, an important step of the GMM is to determine the number of Gaussian components. Further research is needed to better determine the number of Gaussian components required. For the quantification of damage size, the calculation method of MI must be further improved. The environmental influence such as humidity is low in the fatigue test. More aircraft fatigue tests will be performed shortly under a more complex time-varying condition.

\section{Acknowledgment}

This work is sponsored by National Natural Science Foundation of China (Grant No.51921003, Grant No. 51635008), Jiangsu Provincial Key Research and Development Program of China (Grant No. BE2018123), Priority Academic Program Development of Jiangsu Higher Education Institutions of China.

\section{References}

[1] Boller C, Chang FK and Fujino Y. Encyclopedia of structural health monitoring. New York: John Wiley \& Sons, 2009. https://doi.org/10.1002/9780470061626

[2] Yuan S F, Ren Y Q, Qiu L, Mei H F. A multi-response-based wireless impact monitoring network for aircraft composite structures. IEEE Transactions on Industrial Electronics, 2016, 63(12): 7712-7722. https://doi.org/10.1109/TIE.2016.2598529

[3] Su Z, Ye L. Identification of Damage Using Lamb Waves. Springer London, 2009. https://doi.org/10.1007/978-1-84882-784-4

[4] Yuan S F, Zhang J J, Chen J, et al. A uniform initialization Gaussian mixture model-based guided wave-hidden Markov model with stable damage evaluation performance. Structural Health Monitoring, 2019, 18(3):853-868. https://doi.org/10.1177/1475921718783652

[5] Tschope C, Wolff M. Statistical Classifiers for Structural Health Monitoring. Sensors Journal, IEEE, 2009, 9(11):1567-1576. https://doi.org/10.1109/JSEN.2009.2019330

[6] Banerjee S, Qing X P, Beard S, et al. Prediction of Progressive Damage State at the Hot Spots using Statistical Estimation. Journal of intelligent material systems and structures, 2010, 21(6):595-605. https://doi.org/10.1177/1045389X10361632

[7] Qiu L, Fang F, Yuan S F, et al. An enhanced dynamic Gaussian mixture model-based damage monitoring method of aircraft structures under environmental and operational conditions. 
Structural Health Monitoring, 2018:147592171875934.

https://doi.org/10.12783/shm2017/13905

[8] Torkamani S, Roy S, Barkey M E, et al. A novel damage index for damage identification using guided waves with application in laminated composites. Smart Materials \& Structures, 2014, 23(9):095015. https://doi.org/10.1088/0964-1726/23/9/095015

[9] Liberti L, Lavor C, Maculan N, Mucherino A. Euclidean Distance Geometry and Applications. SIAM Review. 2014; 56:3-69. https://doi.org/10.1137/120875909

[10] De Maesschalck R, Jouan-Rimbaud D, Massart DL. The Mahalanobis distance. Chemometrics and Intelligent Laboratory Systems. 2000; 50:1-18. https://doi.org/10.1016/S0169-7439(99)00047-7

[11] You C H, Lee K A, Li H. GMM-SVM Kernel with a Bhattacharyya-Based Distance for Speaker Recognition. Audio Speech \& Language Processing IEEE Transactions on, 2010, 18(6):1300-1312. https://doi.org/10.1109/TASL.2009.2032950

[12] Goldberger J, Gordon S, Greenspan H. An efficient image similarity measure based on approximations of KL-divergence between two Gaussian mixtures// Proceedings Ninth IEEE International Conference on Computer Vision. IEEE, 2003.

https://doi.org/10.1109/ICCV.2003.1238387 